ISSN 1996-1073

www.mdpi.com/journal/energies

Article

\title{
Seasonal Variation of Lipids and Fatty Acids of the Microalgae Nannochloropsis oculata Grown in Outdoor Large-Scale Photobioreactors
}

Martin Olofsson ${ }^{1}$, Teresa Lamela ${ }^{2}$, Emmelie Nilsson ${ }^{1}$, Jean Pascal Bergé ${ }^{3}$, Victória del Pino ${ }^{2}$, Pauliina Uronen ${ }^{4}$ and Catherine Legrand ${ }^{1, *}$

1 Centre for Ecology and Evolution in Microbial model Systems (EEMiS), Linnæus University, 39182 Kalmar, Sweden; E-Mails: martin.olofsson@lnu.se (M.O.); emmelie.nilsson@lnu.se (E.N.)

2 Necton SA, 8700-152 Olhão, Portugal; E-Mails: teresa@necton.pt (T.L.); vdelpino@necton.pt (V.d.P.)

3 Laboratory of Science and Technology of the Marine Biomass (STBM), Ifremer, 44311 Nantes cedex 3, France; E-Mail: jpberge@ifremer.fr

4 Neste Oil, 00095 Espoo, Finland; E-Mail: pauliina.uronen@nesteoil.com

* Author to whom correspondence should be addressed; E-Mail: catherine.legrand@lnu.se; Tel.: +46-480-447309; Fax: +46-480-447305.

Received: 12 March 2012; in revised form: 3 May 2012 / Accepted: 9 May 2012 /

Published: 21 May 2012

Abstract: While focus in oil-producing microalgae is normally on nutrient deficiency, we addressed the seasonal variations of lipid content and composition in large-scale cultivation. Lipid content, fatty acid profiles and mono- di- and triglycerides (MAGs, DAGs, and TAGs) were analyzed during May 2007-May 2009 in Nannochloropsis oculata grown outdoors in closed vertical flat panels photobioreactors. Total lipids (TL) ranged from $11 \%$ of dry weight (DW) in winter to $30 \%$ of DW in autumn. $50 \%$ of the variation in TL could be explained by light and temperature. As the highest lipid content was recorded during autumn indicating an optimal, non-linear, response to light and temperature we hypothesize that enhanced thylakoid stacking under reduced light conditions resulted in more structural lipids, concomitantly with the increase in glycerides due to released photo-oxidative stress. The relative amount of monounsaturated fatty acids (MUFA) increased during autumn. This suggested a synthesis, either of structural fatty acids as MUFA, or a relative increase of C16:1 incorporated into TAGs and DAGs. Our results emphasize the significant role of environmental conditions governing lipid content and 
composition in microalgae that have to be considered for correct estimation of algal oil yields in biodiesel production.

Keywords: biodiesel; microalgae; seasonal variation; Nannochloropsis oculata; large-scale; lipids; fatty acids; light; temperature

\section{Introduction}

The shortages of fossil fuels and increasing demands for reductions in $\mathrm{CO}_{2}$ emissions has led to extensive research regarding alternative renewable $\mathrm{CO}_{2}$-neutral fuel sources. Microalgae as oil-producing organisms and thus a potential feedstock for biodiesel production are well recognized and described [1-4]. The main advantages of microalgae-derived biodiesel are: (1) higher production rates per unit area compared to land grown crops, (2) the possibility to grow on marginal or non-arable land, (3) utilization of seawater and waste water, therefore reducing fresh water use, (4) both $\mathrm{CO}_{2}$ neutral fuel manufacture and $\mathrm{CO}_{2}$ sequestration (5) non-toxic, biodegradable and renewable fuel [3].

Large-scale microalgal cultivation has mainly been studied for the production of live feed (rotifers) in aquaculture [5-9] and for food additive purposes [10,11]. Some studies have investigated lipid accumulation and composition in outdoors cultivation as a function of light and temperature $[5,11,12]$. In recent years, the focus on renewable fuel sources has led to the study of large-scale production of microalgae and lipid accumulation [13-15]. However, relatively few studies have addressed seasonal lipid variation in outdoor large-scale microalgal production.

Microalgae contain primarily polar structural lipids such as phospholipids and glycolipids together with neutral storage lipids such as monoglycerides (MAGs), diglycerides (DAGs) and triglycerides (TAGs), free fatty acids, hydrocarbons and pigments. Polar lipids are confined to membranes of organelles in the cell such as the thylakoid membranes of the chloroplast $[2,16]$. TAGs are neutral lipids mainly stored in vacuoles within the cell $[2,17]$. Most microalgae accumulate very little TAGs during exponential growth but substantial amount of TAGs can accumulate during stationary phase [17-19]. The fatty acid (FA) composition may differ between lipid classes. Predominantly, saturated fatty acids (SAFA) and monounsaturated fatty acids (MUFA) comprise the storage lipid fraction, while polyunsaturated fatty acids (PUFA) largely comprise the structural lipid fraction [2]. The storage lipids in Nannochloropsis sp. was found to consist largely of the SAFA palmitic acid (C16:0) and the MUFA palmitoleic acid (C16:1) [20]. However, Tonon et al. [19] found that 68\% of the TAGs in Nannochloropsis oculata consisted of the PUFA eicosapentaenoic acid (20:5 $\omega 3$, EPA) at the end of stationary phase compared with $8 \%$ during exponential phase.

Several factors may influence algal biomass production; lipid content and composition and thus oil yield of microalgae such as species selection and growth conditions. Under nutrient stress conditions the TL content may increase and more lipids are stored in the form of TAGs and also with a change in FA composition [13,15,21-23]. Salinity [24] and $\mathrm{CO}_{2}$ [14] may also change lipid content and composition. Moreover, TL, FA composition and TAGs formation of microalgae have been reported to vary with light $[5,11,12,16,20,25,26]$ and temperature $[5,11,16,27,28]$. The general notion of these studies is that high light and high temperature generate a more saturated FA composition with 
increased TAGs formation compared to low light and low temperature conditions. Given that light and temperature vary seasonally, these parameters are crucial for understanding lipid accumulation and composition in microalgae. Variations of environmental factors resulting in large seasonal variations of biomass production in outdoor large scale culturing must be taken into account for a sustainable production of algal feedstock for fuels or aquaculture. In addition, the seasonal variation of lipid content and composition within the microalgal cells must also be considered for correct estimations of algal oil yield.

Biodiesel is manufactured using a transesterification process where vegetable or animal TAGs are reacting with an alcohol (typically methanol) to produce an ester then referred to as a fatty acid methyl ester (FAME) and glycerol [1]. Distinct from biodiesel is renewable diesel, such as hydrotreated vegetable oil (HVO) that is chemically the same as petrodiesel, but is derived from a wide range of vegetable sources, although waste animal fats can be used [29]. The hydrogenation of TAGs produces a pure hydrocarbon chain (paraffin) containing no oxygen but small amounts of water, $\mathrm{CO}_{2}$ and propane as by-products [29]. Since biodiesel and HVO production from TAGs requires fatty acids of the right length and characteristic it is essential to determine the fatty acid composition of microalgae. Oxidative stability and combustion quality of diesel fuel in different temperatures are important features in biodiesel production [3] together with similar properties to high value oil and adaptability to industrial production [4]. Important fatty acids for biodiesel production have been proposed to be C16:1, C18:1 and C14:0 in the ratio of 5:4:1 by Klopfenstein [3] depending on oxidative stability, temperature and combustions quality of diesel fuel. Meng et al. [4] suggested a high content of C18:0 and C18:1 in microorganisms to be a prerequisite in biodiesel production due to similar properties of high value oil, oxidative stability and larger potential adaptability in industrial production. C16:0 has also been proposed as a suitable fatty acid in renewable diesel production. It is also a common fatty acid in microalgae as well as in oil from higher plants [30-32]. However, refining oil through hydrogenation would account for more flexibility since the process allows removal of double bonds of unsaturated fatty acids to some degree. Moreover, besides TAGs other glycerides, mono- and diglycerides (MAGs, DAGs) and free fatty acids (FFA) can be used in the process [33].

The aim of this study was to monitor the seasonal variation of: (i) lipid content, (ii) fatty acid (FA) profile and (iii) neutral lipid classes, such as glycerides (MAGs, DAGs, TAGs) and FFA in relation with light and temperature for the marine Eustigmatophyte $N$. oculata grown outdoors in large-scale photobioreactors. Total lipids (TL), FA profiles and neutral lipid classes were measured in N. oculata cultures between May 2007 and May 2009. Nannochloropsis oculata is a marine microalgae and one of 6 species within the genus Nannochloropsis belonging to the class of Eustigmatophyceae. The genus is widely used in aquaculture [5-9] due to its relatively high growth rate, resistance to mixing and contamination together with high nutritional values and a high lipid content $[7,8,20]$. These characteristics also fit the needs of the biofuel industry. 


\section{Results and Discussion}

\subsection{Total Lipids (TL)}

Algal biomass concentration ranged $1.10-2.32 \mathrm{~g} \cdot \mathrm{L}^{-1}$. The seasonal variation of lipid content (TL) in Nannochloropsis oculata grown outdoors ranged 10\%-30\% DW (Figure 1). In winter and spring TL ranged $11 \%-24 \%$ and $13 \%-22 \%$ of DW and indicated both a variation between years (2008 and 2009) and during the season. However, no variation could be found between winter and spring values. During summer and autumn, the lipid content was between $20 \%$ and $30 \%$ of DW. These levels are comparable to those obtained with the same algal genus in controlled laboratory $[13,14,21]$ and outdoors studies $[5,13,15]$.

Figure 1. Seasonal variation of total lipids (TL, filled circles) of N. oculata in relation to temperature (filled diamonds) and light in the form of total global radiation (TGR, open triangles) during 2007-2009. Horizontal bars denote periods when cooling of photobioreactors may occur (set point $25^{\circ} \mathrm{C}$ ).

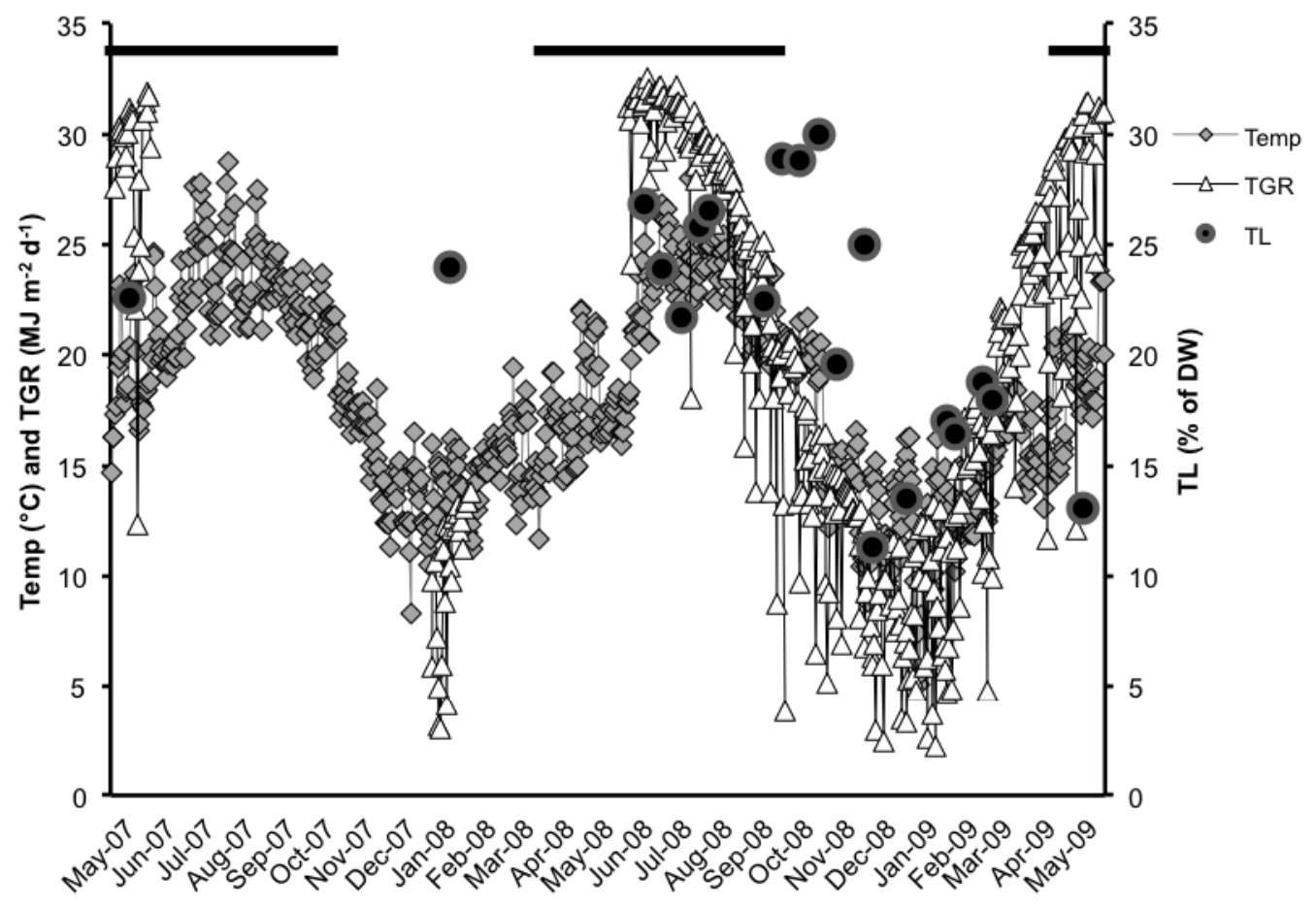

Many studies underline the important role of light and temperature in algal lipid synthesis and accumulation [5,16,20,23,27,28,34]. In general, high lipid content was related to high light, measured as total global radiation (TGR), and high temperature, while lower lipid content was found at low TGR and low temperature. On the other hand, the peak in lipid content in October 2008 coincided with the decrease in TGR and temperature during autumn. During winter the lipid content decreased to as low as $11 \%$ of DW (December 2008). In late February the TL increased to $19 \%$ of DW. Thereby, a variation between winter low and high during one season could be found. A variation between two spring seasons was also found as TL in May 2007 was 22\% and TL in May 2009 was 13\%. However, no variation was recorded between the winter and spring samples of 2007 and 2008. TL of the first two samples (May 2007 and Jan 2008) was 22\% and 24\% of DW respectively (Figure 1). These variations 
suggested both intra-seasonal and inter-seasonal variations. Whether these contrasting results all can be attributed to environmental conditions remains uncertain. This reveals the importance of higher frequency monitoring of outdoor algal cultures to implement current productivity models. Our results revealed that under optimal growth conditions in closed photobioreactors up to $50 \%$ of the TL variation could be explained by both TGR and temperature (multiple linear regression, $y_{\mathrm{TL}}=2.64+1.70 x_{\mathrm{Temp}}-0.65 x_{\mathrm{TGR}}, R^{2}=0.51, p=0.0023$ ). Lipids (TL) tend to accumulate more in the cells at high or photoinhibiting irradiance than under limiting conditions for photosynthesis [5,20,23]. In summer, $N$. oculata responded to high light by accumulating more TL than in winter. However, elevated TL levels in the autumn (September-October) did not fit into the linear model as for the other seasons. Temperature alone explained 38\% of the variation (simple linear regression, $y_{\mathrm{TL}}=8.73+0.72 x_{\mathrm{Temp}}, R^{2}=0.38, p=0.0039$ ). The strong positive relationship between temperature and TL over the seasons is in contrast with previous studies reporting either a weak or a negative relationship between the two variables for various algal species [34]. Lipids accumulated more in the green algae Dunaliella cells grown at low $\left(12^{\circ} \mathrm{C}\right)$ compared to high $\left(30^{\circ} \mathrm{C}\right)$ temperature, and even more when cells responded to a sudden shift from $30^{\circ} \mathrm{C}$ to $12{ }^{\circ} \mathrm{C}$ [27]. The authors concluded that lower temperature led to the enhancement of structural lipids to facilitate cell membrane fluidity. This process is known to occur generally in higher plant cells when temperature decreases [35]. In our study, it is unlikely that summer temperatures dropped to levels below the cell membrane fluidity threshold. In winter, this threshold was reached on few occasions but TL levels remained low as the cell demand in energy possibly prevented lipid storage.

Culture density affects light availability to each cell in the culture and may also play a role in governing lipid accumulation. However, the biomass range (1.1-2.3 $\mathrm{g} \cdot \mathrm{L}^{-1}$ of dry weight) was relatively constant during run time for all seasons, and the light path $(5 \mathrm{~cm})$ in the flow-through vertical photobioreactor was relatively short to ensure optimum light availability. Thus cell density is unlikely to account for major differences in cellular lipid content. The $50 \%$ of the variation in TL not accounted for by the multiple linear regression may still be attributed to other factors (e.g., photon utilization efficiency, photoinhibition, temperature fluctuations) in combination with a non-linear response to environmental conditions. The autumn in Algarve (South of Portugal) is often warm and dry with sunny days and cool nights. Short-term changes in day length, photoperiod and light intensity may explain the high TL levels in the autumn. Between July and September, sun-hours and light intensity drop relatively faster $(3 \mathrm{~h})$ than temperature during the day. This relative fast decrease in effective day length (photoperiod $\mathrm{x}$ intensity) could result in lower photosynthetic rate followed by a rearrangement of the photosynthetic apparatus to achieve a more efficient light harvesting and utilization capacity. The algal cell adapts with a significant enlargement of thylakoid membranes, the enhancement of stacked thylakoid membranes and an increased synthesis of light harvesting complexes $[25,36]$. When facing a drastic change in light intensity, algal cells achieve the support of this new membrane structure by increasing lipid synthesis over a short time (2-3 days) [25], hence increasing thylakoid membranes fluidity. Nannochloropsis grown in tubular photobioreactors in the South of Europe (Italy) accumulated highest amounts of fatty acids during days with high irradiance followed by low temperature nights [12]. In the Autumn 2008, at our experimental site in Algarve the difference in temperature between a warm day $\left(25^{\circ} \mathrm{C}\right)$ and a cool night varied in the air by $10-20{ }^{\circ} \mathrm{C}$ and in the water by $5-10{ }^{\circ} \mathrm{C}$. Diurnal temperature variation combined to high irradiance likely 
contributed to the high and relatively stable levels of TL in September-October. Future work should be directed to understanding short-term fluctuations obtained from outdoors cultivation studies to improve predictions of the potential algal production yield over a year. High levels of TL in the autumn may be combined with other mechanisms of lipid induction (e.g., nitrogen limitation) to improve biomass and lipid yields in outdoors production units.

\subsection{Neutral Lipid Classes}

The neutral lipid classes investigated apart from triglycerides (TAGs) were monoglycerides (MAGs), diglycerides (DAGs), free fatty acids (FFA) and oligomers (Figure 2). TAGs ranged $10 \%-31 \%$ of total neutral lipids (TNL) with the highest proportion of TAGs found during late summer (23\%-31\% of TNL). No significant relationship was found between TAGs and temperature or light (simple and multiple regression, $p>0.05)$. DAGs fluctuated with the lowest fraction in winter $(32 \%)$ and the highest in summer (56\%) and autumn (50\%), during the peak in TL (Figure 1).

Figure 2. The relative proportions of some lipid classes (shown by stacked columns) in N. oculata during the period of June 2008 to March 2009 together with temperature (solid line) and total global radiation (dashed line). In the stacked columns from above: Free FA (grey), monoglycerides (MAGs, dark grey), diglycerides (DAGs, medium grey), Triglycerides (TAGs, black) and oligomers (light grey).

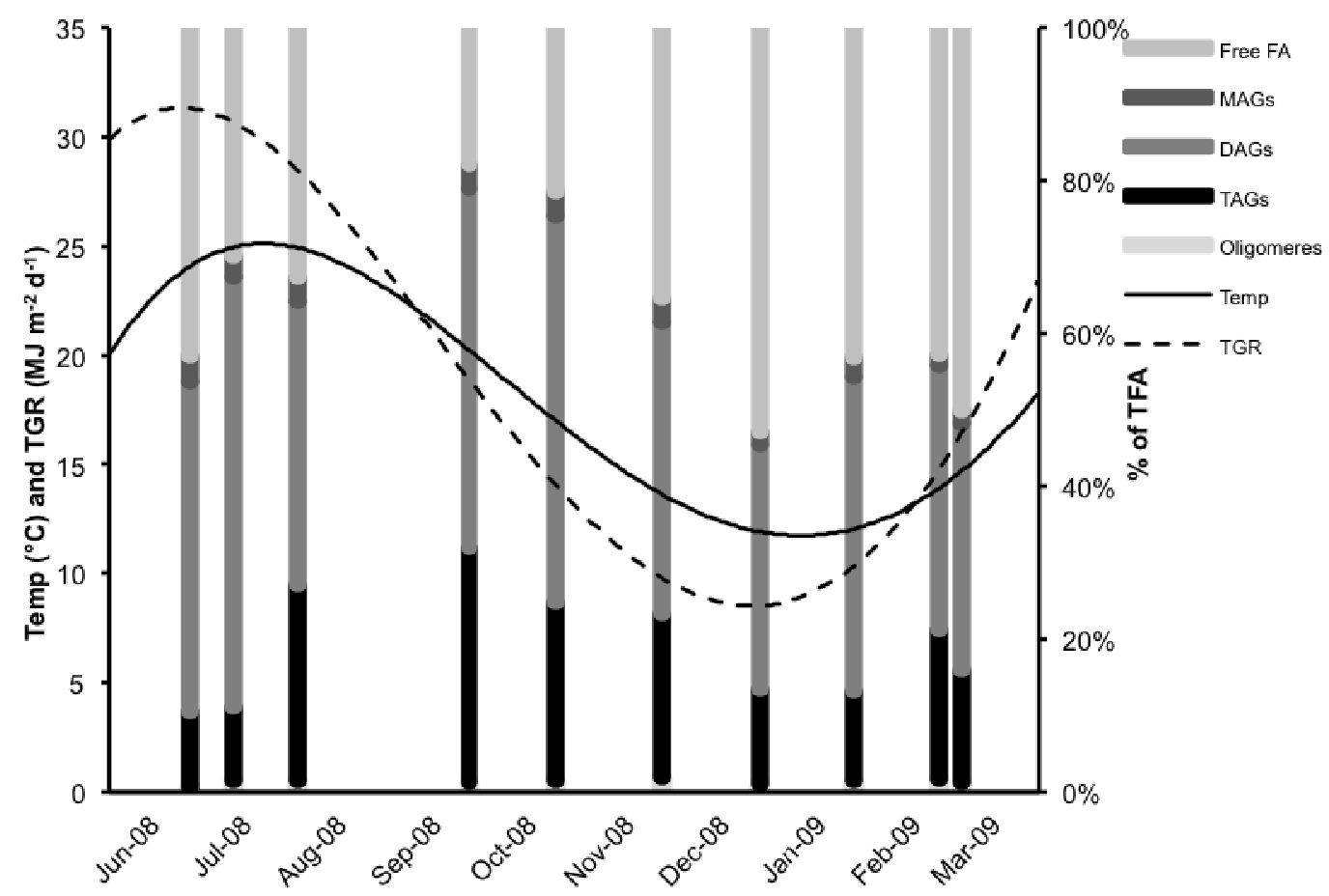

A positive significant relationship between DAGs and temperature was found (simple regression, $\left.y_{\mathrm{DAG}}=21.6+1.08 x_{\mathrm{Temp}}, R^{2}=0.46, p=0.0323\right)$. MAGs only constituted a minor part $(<3.5 \%)$. The total fraction of glycerides (mono- di- and triglycerides) together comprised $46 \%-80 \%$ of TNL with the highest recording in late September. Total glycerides were significantly related with both light and temperature. However, temperature explained most of the variation in total glycerides (simple regression, $\left.y_{\text {TotGlyc }}=26.0+1.97 x_{\text {Temp }}, R^{2}=0.75, p=0.0012\right)$. Free fatty acids $(\mathrm{FFA})$ ranged $18 \%-53 \%$ 
of TNL with maximum value in winter. Temperature, significantly, explained the variation in FFA (simple regression, $y_{\mathrm{FFA}}=72.9-1.08 x_{\mathrm{Temp}}, R^{2}=0.75, p=0.0012$ ). The fraction of lipids in the form of oligomers was below $2 \%$ in all of the samples.

Although over 40000 microalgal species have been identified, few species have been screened for neutral lipid composition (glycerides and free fatty acids) relevant for biofuel production and FA profiles in the literature [37]. For the oleaginous Nannochloropsis widely used in large-scale production, we are aware of little more than ten references, including our study, reporting such data $[2,5,8,12,16,17,19-21,26,38]$. Consequently our study provides valuable information on how individual algal neutral lipids respond differently to environmental variables such as ambient temperature and sunlight under non-limiting nutrient conditions. Temperature explained up to $75 \%$ of the seasonal variation of both glycerides and FFA. However, total glycerides increased during late summer-autumn in relation to FFA, concomitantly with the rise in TL indicating a significant glyceride synthesis.

TAGs are storage lipids usually synthesized under adverse environmental conditions (low light and temperature) or photo-oxidative stress [2]. TAGs serve as electron sinks during photo-oxidative stress preventing reactive oxygen species (ROS) damage to membrane lipids and proteins. During summer, clouds do not reflect sunlight and shortwave radiations (UV) increase, possibly triggering photoinhibition. As pointed out in laboratory studies, Nannochloropsis cells would synthesize more TAG under photoinhibition conditions [20]. Considering that certain ROS are strong oxidizing agents, which can directly attack neutral lipids, we suggest that both an over reduced electron chain and the presence of ROS may have prevented the accumulation of newly synthesized TAGs. Moving towards autumn, photoinhibition is relaxed and TAGs accumulated again. Our data point out that palmitoleic acid (C16:1) and MUFA contributed the most to the increase of both neutral and total lipids. Similarly, higher levels of $\mathrm{C} 16: 1$ were found in Nannochloropsis cultures grown at $20^{\circ} \mathrm{C}$ compared to $30{ }^{\circ} \mathrm{C}$ [16] suggesting that the cooler autumn temperatures and large diurnal variation may favor the allocation of suitable FA for biodiesels to TAGs. Towards the winter, total glycerides, of which TAGs and DAGs, decreased by more than $30 \%$ compared to the autumn values. TAGs are also energy reserve molecules and were used during sub-optimal growth conditions.

In contrast to laboratory studies where the TAG:DAG ratio in Nannochloropsis varies from 2:1 to $4: 1$ [20], ratios of $1: 2$ to $1: 4$ were measured during the study period. Differences in composition may vary due to growing conditions (laboratory vs. outdoors), analytical method (GC vs. GPC) and the origin of the strain. Revealing the impact of light on algal metabolism indoors is hardly comparable to outdoors studies where ambient conditions are changing at both short and long temporal scales.

\subsection{Fatty Acid (FA) Profile}

FAs suitable for biodiesel production such as myristic acid (C14:0), palmitic acid (C16:0), palmitoleic acid (C16:1), stearic acid (C18:0) and oleic acid (C18:1) constituted $45 \%-78 \%$ of total fatty acids (TFA) except in the two first samples (Figure 3). Palmitic acid (C16:0) and palmitoleic acid (C16:1) were the most frequent fatty acids encountered in N. oculata profiles (Table 1). 
Figure 3. Seasonal variation of desired FA suitable for fuel production in N. oculata during 2007-2009 in percentage of total FA.

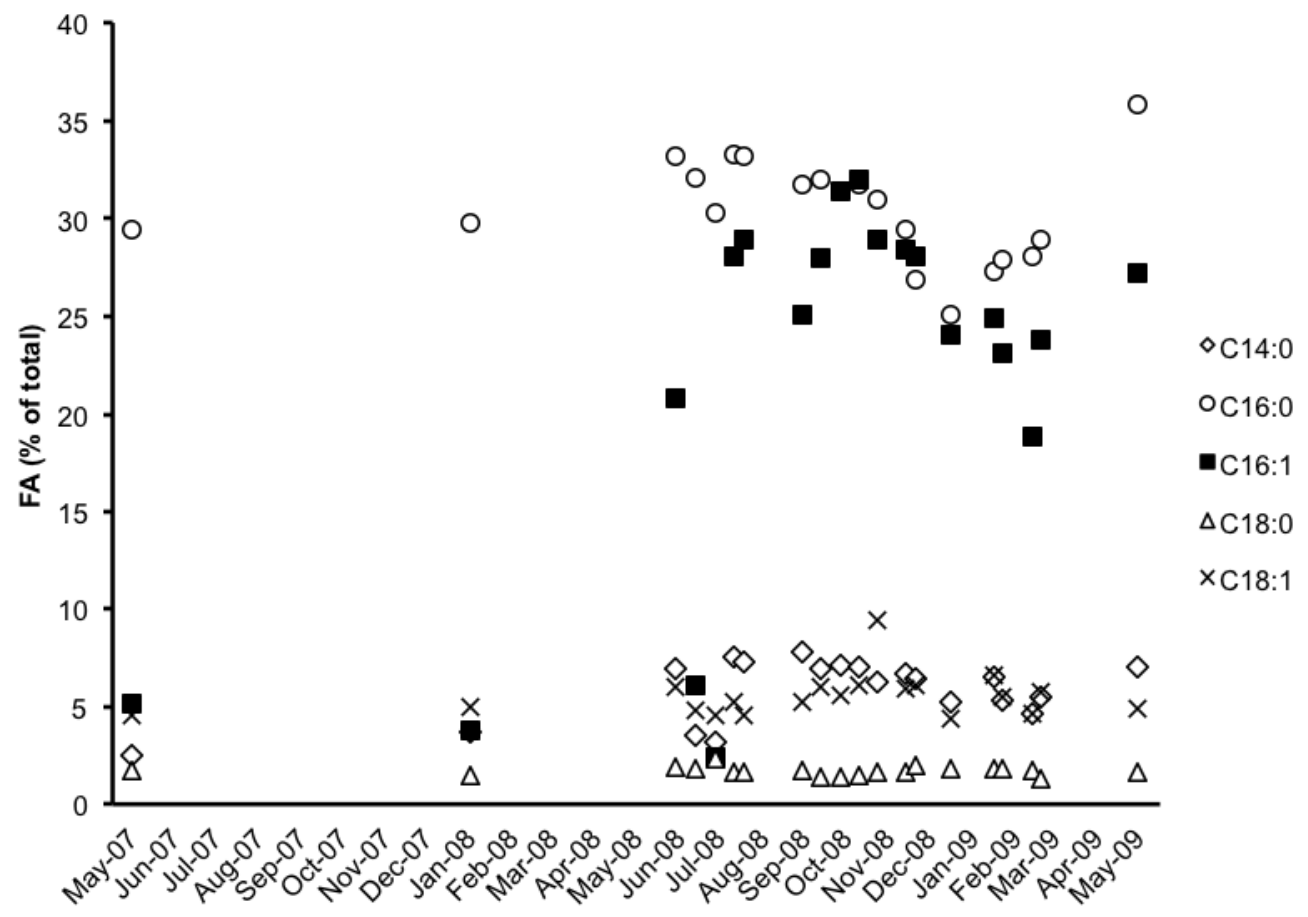

These FA contributed $30 \%-60 \%$ of the TFA in all samples (Figure 3). Myristic acid (C14:0) and stearic acid (C18:0) were relatively stable throughout the study period. C16:1 levels increased from winter to summer-autumn, when the highest values were recorded. C18:0 never reached above 2\% of TFA. C18:1 only went through minor changes over the examined period. Odd values of C14:0 and C16:1 ( $<10 \%$ of TFA) were observed in late June and mid July when temperature was above $30{ }^{\circ} \mathrm{C}$ (Figures 1 and 3). TGR (simple regression, $y_{\mathrm{C} 16: 0}=23.1+0.41 x_{\mathrm{TGR}}, R^{2}=0.54, p=0.0002$ ) and temperature (simple regression, $y_{\mathrm{C} 16: 0}=26.0+0.25 x_{\mathrm{Temp}}, R^{2}=0.55, p=0.0002$ ) explained equally well the variation in C16:0. No significant relationship was found between the total amounts of suitable FA and TGR or temperature due to odd values of C16:1 (<10\% of TFA) in May 2007, January 2008 and late June-mid July 2008. Suitable FA for biofuel production followed approximately the TL variation in 2008-2009. In general, high concentrations of suitable FA corresponded to high levels of TL in the autumn, mainly depending on the increase of C16 and C18 FA. Conversely, in May 2009 the suitable FA constituted a significant part of the TL (Figure 3) when the lipid content was low (Figure 1).

Table 1. Seasonal variation in FA profiles of $N$. oculata (percentage of TL) during 2008-2009.

\begin{tabular}{cccccccccc}
\hline FA & 13-Jun-08 & 27-Jun-08 & 11-Jul-08 & 25-Jul-08 & 1-Aug-08 & 12-Sep-08 & 26-Sep-08 & 10-Oct-08 & 24-Oct-08 \\
\hline C14:0 & 6.9 & 3.5 & 3.2 & 7.5 & 7.3 & 7.8 & 6.9 & 7.1 & 7 \\
C15:0 & 0.7 & 0.7 & 0.8 & 0.7 & 0.7 & 0.7 & 0.6 & 0.7 & 0.7 \\
C16:0 & 33.2 & 32.1 & 30.3 & 33.3 & 33.2 & 31.7 & 32 & 31.4 & 31.7 \\
C16:1 $\omega 7$ & 20.8 & 6.1 & 2.4 & 28.1 & 28.9 & 25.1 & 28 & 31.4 & 32 \\
C16:2 $\omega 4$ & 2.1 & 6.5 & 6.7 & 0.7 & 0.7 & 0.9 & 0.6 & 0.6 & - \\
C16:3 $\omega 4$ & 1.4 & 4.4 & 6.7 & - & - & 0.5 & - & - & - \\
C18:0 & 1.9 & 1.8 & 2.3 & 1.6 & 1.6 & 1.7 & 1.4 & 1.4 & 1.5 \\
C18:1 $\omega 9$ & 5.3 & 3.8 & 3.1 & 4.7 & 4.1 & 4.7 & 5.6 & 5.2 & 5.7 \\
\hline
\end{tabular}


Table 1. Cont.

\begin{tabular}{cccccccccc}
\hline FA & 13-Jun-08 & 27-Jun-08 & 11-Jul-08 & 25-Jul-08 & 1-Aug-08 & 12-Sep-08 & 26-Sep-08 & 10-Oct-08 & 24-Oct-08 \\
\hline $\mathrm{C} 18: 1 \omega 7$ & 0.7 & 1 & 1.4 & 0.5 & 0.4 & 0.5 & 0.4 & 0.4 & 0.4 \\
$\mathrm{C} 18: 2 \omega 6$ & 8.4 & 24 & 24.8 & 2.9 & 2.4 & 4.3 & 2.9 & 2.6 & 2.5 \\
$\mathrm{C} 18: 3 \omega 4$ & - & - & - & - & 0.5 & 0.3 & 0.6 & - & - \\
$\mathrm{C} 18: 3 \omega 3$ & 3.9 & 11.9 & 16.3 & 0.4 & 16.3 & 1.7 & - & - & - \\
$\mathrm{C} 20: 4 \omega 6$ & 3.7 & 1.1 & 0.4 & 5.4 & 5.6 & 5.6 & 5.9 & 5.7 & 5.6 \\
$\mathrm{C} 20: 5 \omega 3$ & 11.1 & 2.4 & 0.8 & 14.2 & 14.7 & 14.4 & 15.1 & 13.3 & 14.7 \\
$\mathrm{C} 22: 3 \omega 3$ & - & 0.6 & 0.8 & - & - & - & - & - & - \\
\hline FA & $\mathbf{7 - N o v - 0 8}$ & $\mathbf{2 8 - N o v - 0 8}$ & $\mathbf{5 - D e c - 0 8}$ & $\mathbf{3 0 - D e c - 0 8}$ & $\mathbf{3 0 - J a n - 0 9}$ & $\mathbf{6 - F e b - 0 9}$ & $\mathbf{2 7 - F e b - 0 9}$ & $\mathbf{6 - M a r - 0 9}$ & $\mathbf{1 5 - M a y - 0 9}$ \\
\hline $\mathrm{C} 14: 0$ & 6.2 & 6.7 & 6.4 & 5.2 & 6.5 & 5.3 & 4.6 & 5.5 & 7 \\
$\mathrm{C} 15: 0$ & 0.7 & 0.7 & 0.7 & 0.7 & 0.8 & 0.7 & 0.7 & 0.7 & 0.7 \\
$\mathrm{C} 16: 0$ & 31 & 29.4 & 26.9 & 25.1 & 27.3 & 27.9 & 28.1 & 28.9 & 35.8 \\
$\mathrm{C} 16: 1 \omega 7$ & 28.9 & 28.4 & 28.1 & 24.1 & 24.9 & 23.1 & 18.9 & 23.8 & 27.2 \\
$\mathrm{C} 16: 2 \omega 4$ & 0.8 & - & 0.9 & 1.2 & 1.2 & 1.3 & 1.3 & 1.1 & - \\
$\mathrm{C} 16: 3 \omega 4$ & - & - & 1.1 & 2.4 & 1.4 & 3 & 4.2 & 1.7 & - \\
$\mathrm{C} 18: 0$ & 1.6 & 1.6 & 2 & 1.8 & 1.8 & 1.8 & 1.7 & 1.3 & 1.6 \\
$\mathrm{C} 18: 1 \omega 9$ & 8.9 & 5.5 & 5.5 & 3.8 & 6 & 4.8 & 3.9 & 5.1 & 4.5 \\
$\mathrm{C} 18: 1 \omega 7$ & 0.5 & 0.4 & 0.6 & 0.6 & 0.6 & 0.7 & 0.7 & 0.6 & 0.4 \\
$\mathrm{C} 18: 2 \omega 6$ & 3.3 & 3.5 & 4.8 & 7.8 & 5.9 & 7.4 & 12.1 & 7.3 & 2.9 \\
$\mathrm{C} 18: 3 \omega 4$ & - & - & - & - & - & - & - & - & - \\
$\mathrm{C} 18: 3 \omega 3$ & - & 1.1 & 2.6 & 5.6 & 3.2 & 6.7 & 10.5 & 4.2 & - \\
$\mathrm{C} 20: 4 \omega 6$ & 5 & 6.3 & 6.1 & 6 & 4.8 & 3.8 & 3.2 & 4.3 & 4.3 \\
$\mathrm{C} 20: 5 \omega 3$ & 13.1 & 16.4 & 14.5 & 15.8 & 15.6 & 13.4 & 10 & 15.3 & 15.6 \\
$\mathrm{C} 22: 3 \omega 3$ & - & - & - & - & - & - & - & - & - \\
\hline
\end{tabular}

In N. oculata FA profiles, Saturated fatty acids (SAFAs) and Unsaturated FA (MUFAs and PUFAs) ranged $35 \%-40 \%$ and $60 \%-65 \%$ of TFA, respectively (Figure 4 ). The profiles revealed seasonal variations in lipid composition as positive significant relationships were found between SAFAs and temperature (simple regression, $y_{\mathrm{SAFA}}=31.7+0.38 x_{\mathrm{Temp}}, R^{2}=0.32, p=0.0088$ ) and between SAFAs and TGR (simple regression, $y_{\mathrm{SAFA}}=34.2+0.25 x_{\mathrm{TGR}}, R^{2}=0.35, p=0.0063$ ). In addition, monounsaturated fatty acids (MUFAs) and polyunsaturated fatty acids (PUFAs) changed, even when there were only minor differences in TL (i.e., spring 2007 vs. winter 2008 in Figure 1). The MUFAs (C16:1 and (C18:1) increased during summer-autumn at the expenses of PUFAs that decreased during the same period (Figure 4). No significant relationships were found between neither MUFAs or PUFAs and temperature or between MUFAs or PUFAs and TGR. Up to 35\% of the variation in SAFA could be explained by a linear function of TGR while the variation in MUFAs and PUFAs indicated a non-linear response to light and temperature. In outdoors Pavlova viridis cultures, PUFAs increased with decreasing light and temperature while TL was constant [11]. Overall PUFAs in our study increased in winter, when light intensity was low, as reported previously $[5,11,20,26]$. While MUFAs were high in the autumn possibly as a result of more C16:1 allocated to TAGs, PUFAs (mainly C16:3 $\omega 4$ and $\mathrm{C} 18: 3 \omega 3$ ) decreased, potentially converted into $\mathrm{C} 16: 1$ and $\mathrm{C} 18: 1$. Both $\mathrm{C} 18: 3 \omega 3$ and $\mathrm{C} 18: 2$ $\omega 6$ increased in winter at lower light intensity and temperature as expected. While only minor changes 
in C20:5 13 (Eicosapentaenoic acid, EPA) were observed in our study, an increase of EPA was observed in light limited Nannochloropsis cells [5,20,26].

Figure 4. Representation of saturated (SAFA), monounsaturated (MUFA) and poly-unsaturated (PUFA) fatty acids of $N$. oculata in percentage of total fatty acids (TFA), obtained from the fatty acid profiles determined from seasonal samples during 2007-2009.

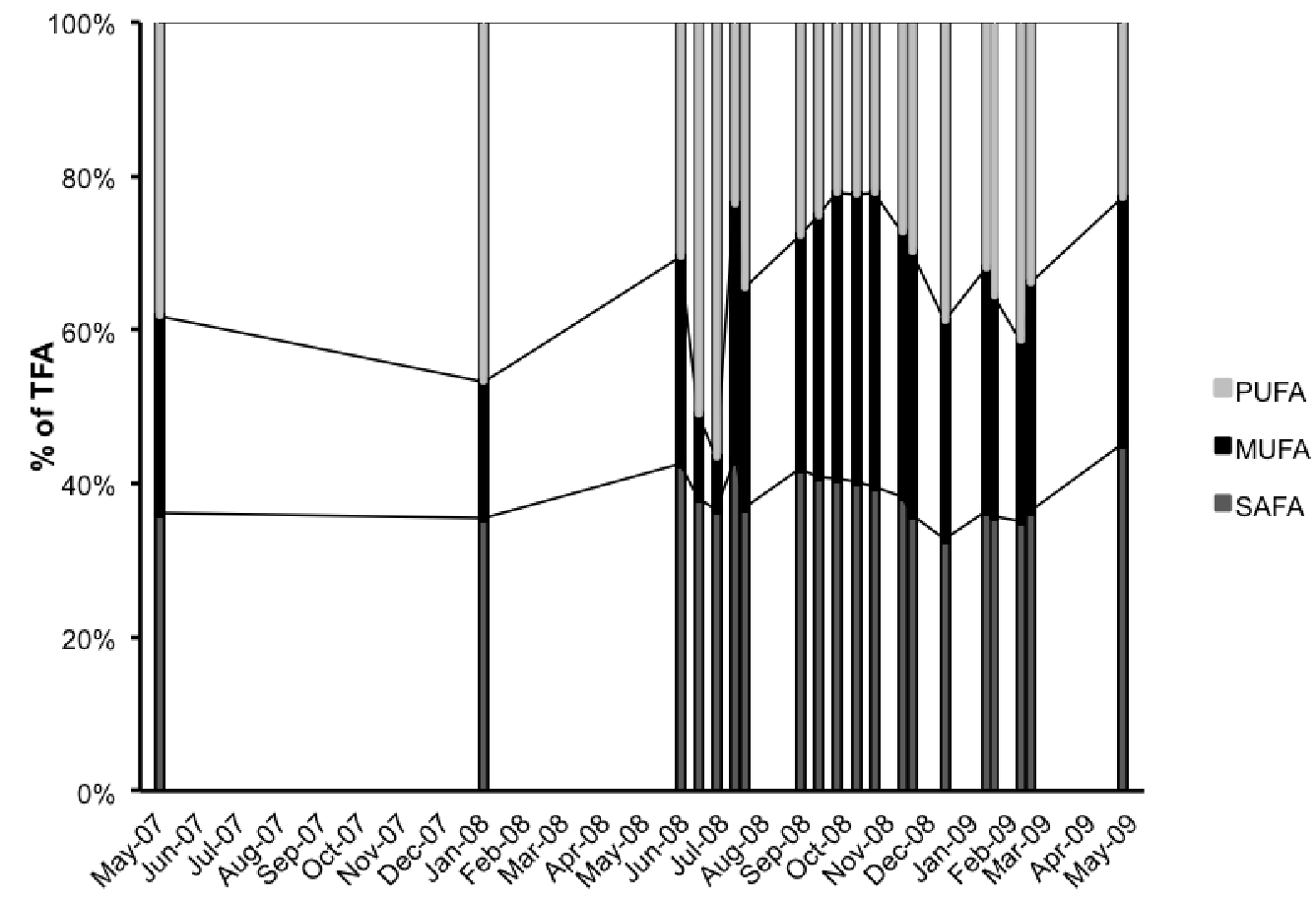

Temperature affects the FA composition of microalgae [27,28,34]. The degree of unsaturation in fatty acids has usually a negative relationship with temperature [39] but was not significant in our study, possibly due to the fact that temperature only explained $38 \%$ of the TL variation. At higher temperature, PUFAs in various microalgae decreased, while SAFAs increased probably due to the fluidity of cell membranes [34]. Most reports including the present study show a tendency for microalgae to form a greater deal of PUFAs in response to low temperature [11,16,27,28]. However, these results also disclose species-specific behaviour [28,34]. During a temperature shift experiment $\left(30^{\circ} \mathrm{C}\right.$ to $\left.12{ }^{\circ} \mathrm{C}\right)$ the green algae Dunaliella salina responded with a decrease of SAFA and an increase of MUFA and PUFA [27]. In contrast, based on 8 marine microalgae, Thompson et al. [28] suggested that the synthesis of short-chained energy rich SAFA increased as a result of a higher energy demand in response to low temperature. This phenomenon may in some species obscure any increase of membrane unsaturation at low temperature. This seemed not to be the case in our study, but the limited number of outdoors studies with scaled up algal cultures over several seasons, limits the degree of generalization.

The seasonal variation of the ratio saturated to unsaturated FA (sat/unsat) is shown in Figure 5. Simple regressions (TGR: $y_{\text {sat/unsat }}=0.54+0.005 x_{\mathrm{TGR}}, R^{2}=0.2, p=0.0455$ and temperature: $\left.y_{\text {sat/unsat }}=0.48+0.008 x_{\text {Temp }}, R^{2}=0.2, p=0.0481\right)$ suggested that light and temperature as single factors explained the change in lipid composition equally well $(20 \%$ each). Thus there was a positive significant relationship between the ratio saturated to unsaturated FA and temperature or solar 
radiation, i.e., summer conditions generated more saturation of FA while winter conditions resulted in less saturation.

Figure 5. Seasonal variation in the saturated to unsaturated ratio (filled squares) of fatty acids (FA) in N. oculata together with temperature (closed diamonds) and total global radiation (TGR, open triangles) during 2007-2009. Horizontal bars denote periods when cooling of photobioreactors may occur (set point $25^{\circ} \mathrm{C}$ ).

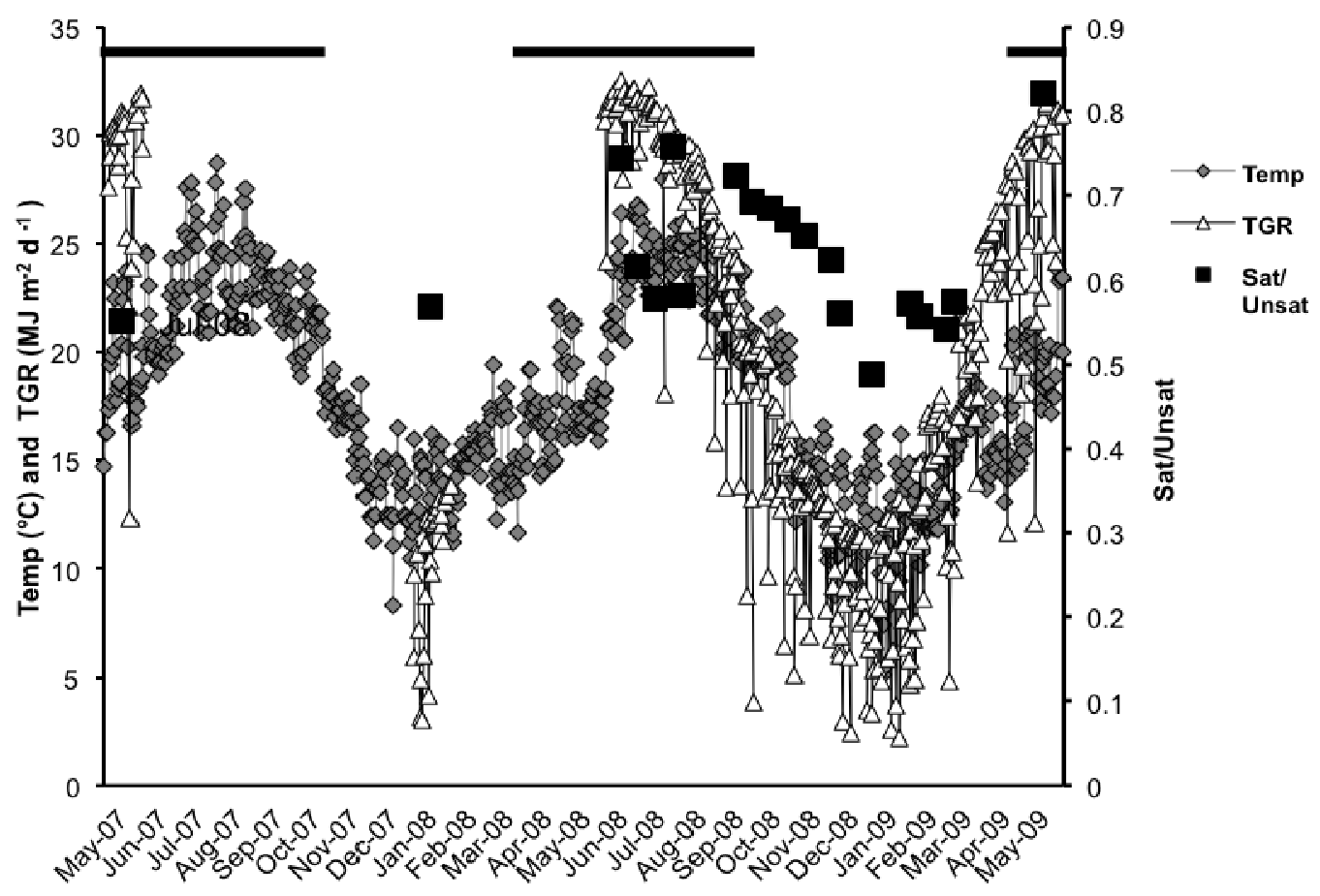

In the autumn, the high degree of saturation diverged from the statistical models similarly to the amount of TL. The synthesis of short-chained FA in response to lower temperature was unlikely, as the average autumn temperatures in Algarve $\left(>20^{\circ} \mathrm{C}\right)$ would not cause a noticeable increase in cellular energy demand. Since lipid saturation was high during autumn, the decrease in light did not result in synthesis of more structural and unsaturated lipids and it is plausible that the reorganization and enlargement of the photosynthetic apparatus lead to the production of more SAFAs and MUFAs at lowered light levels. As discussed above, we hypothesize that diurnal variations in light and temperature in the autumn (warm day, cool night) played a role in the elevated TL, TAGs, SAFAs and MUFAs. Since the proposed best suitable FA in algal biofuel production consisted of SAFAs and MUFAs, autumn conditions seemed to favor optimal lipid accumulation in N. oculata. Compositional profiles can vary greatly from one algal species to the next, depend on growth conditions, and have high uncertainty due to the limited number of published work [37]. Within the genus Nannochloropsis FA profiles have been published for about 10 strains by different research groups, and are in general similar. The proportion of PUFAs of each strain may be the most diverse though. The strains of N. oculata CS-179 [5,38,40], N. oculata CS-216, Nannochloropsis sp. CS-246 [40], Nannochloropsis sp. "Cephalonia Fisheries, Greece" [2,16,20,26] and N. oculata NAOC [8] have consistently higher EPA levels than N. oculata "commercial strain Necton, Portugal" (our study), N. salina CS-190 [40], N. oculata CCAP 849/1 [19], Nannochloropsis sp. "Zmora-IOLR, Israel" [12] and Nannochloropsis 
sp. (origin missing) [15]. Due to oxidative instability of polyunsaturated fatty acids (PUFAs) and the fact that microalgal lipids, in general, contain a great deal of PUFA, these fatty acids must be considered in the efficiency of microalgae for biodiesel production [3]. According to the European Standard for Biodiesel EN 14214 [41] the limit for linolenic acid (C18:3 $\omega 3)$ is $12 \%$ of TL and fatty acids with four or more double bonds should only be $1 \%$ of TL. $N$. oculata fulfills the criteria for the linolenic acid. However, C20:4 $\omega 6$ and C20:5 $\omega 3$ represented the major part of the PUFA fraction in outdoors cultivation and ranged $15 \%-20 \%$ (Table 1, [15]) to $35 \%-40 \%$ [5] of the total FA. In Algarve, seasonal variation of PUFAs showed summer peaks in late June till mid-July that are not likely due to oxidation as linoleic (C18:2) and linolenic (C18:3) acids increased, and remained unexplained. At refining steps in production of FAME biodiesel, the disadvantage of high content of PUFA can be partly managed by catalytic hydrogenation of the oil [1]. As for the HVO, the PUFA fraction will pose a minor problem [29]. It has been suggested that oil with high content of PUFA may be mixed with vegetable, or other microalgal oil to achieve the appropriate composition [15]. However, a much more appealing concept would be the utilization of PUFA as high value fatty acids for food and feed in the summer season perhaps, which probably is necessary for making microalgal fuel production environmentally and economically sustainable [42].

\section{Experimental Section}

N. oculata (commercial strain, Necton) was grown outdoors in 1200-1800 L closed vertical flat panel flow-through photobioreactors at Necton's facility in Algarve (Olhão, Portugal). The water used for the semi-continuous cultures was pumped from ground seawater (salinity 35), filtered $(1 \mu \mathrm{m})$ and pasteurized $\left(60^{\circ} \mathrm{C}\right)$. Nutrients were added as NutriBloom medium (Necton's commercial and industrial culture medium recipe) to reach nitrate levels of $2 \mathrm{mM}$ and phosphate levels of $0.1 \mathrm{mM}$ (final concentration in circulating cultures, $\mathrm{N}: \mathrm{P}=20$ ). Original NutriBloom medium contains $2 \mathrm{M} \mathrm{NaNO}_{3}, 100 \mathrm{mM} \mathrm{KH}_{2} \mathrm{PO}_{4}, 20 \mathrm{mM} \mathrm{FeCl}, 20 \mathrm{mM}$ EDTA-Na, $1 \mathrm{mM} \mathrm{ZnCl} 2,1 \mathrm{mM} \mathrm{ZnSO}-\mathrm{H}_{2} \mathrm{O}$, $1 \mathrm{mM} \mathrm{MnCl}_{2} \cdot 4 \mathrm{H}_{2} \mathrm{O}, 0.1 \mathrm{mM} \mathrm{Na} 2 \mathrm{MoO}_{4} \cdot 2 \mathrm{H}_{2} \mathrm{O} 0.1 \mathrm{mM} \mathrm{CoCl}{ }_{2} \cdot 6 \mathrm{H}_{2} \mathrm{O}, 0.1 \mathrm{mM} \mathrm{CuSO} \cdot 5 \mathrm{H}_{2} \mathrm{O}, 6.4 \mathrm{mM}$ EDTA-Na, 2 mM MgSO $4-7 \mathrm{H}_{2} \mathrm{O}$. The nitrate concentration was monitored daily (data not shown) and fresh NutriBloom medium was added to maintain nitrate concentration at $2 \pm 0.2 \mathrm{mM}$ and constant $\mathrm{N}: \mathrm{P}=20$ to ensure sufficient nutrient availability. When culture biomass reached $1 \mathrm{~g} \cdot \mathrm{L}^{-1}$ of dry weight $15 \%-20 \%$ of the biomass was removed twice a week during commercial harvest. The photobioreactors were replenished with filtered seawater along with NutriBloom medium at original nutrient concentrations $(\mathrm{N}: \mathrm{P}=20)$. The semi-continuous cultivation system has an online set point for $\mathrm{pH}$. When $\mathrm{pH}$ reached $8.5 \mathrm{CO}_{2}$ was injected into the system at 2 bar pressure. When culture temperature reached $25{ }^{\circ} \mathrm{C}$ the reactor panels were cooled with water sprinklers. Occasionally, culture temperatures reached $30^{\circ} \mathrm{C}$ during summer.

The semi-continuous cultures were circulated in stable conditions (steady-state) for 4-6 weeks after inoculation in each season. Samples for total lipid (TL), fatty acid profile (FA) and lipid classes were collected during steady-state of commercial production in 2007-2009, as follows: spring = 3 samples, summer $=5$ samples, autumn $=6$ samples and winter $=6$ samples. At the time of commercial harvest (biomass concentration ranged from 1.1 to $2.3 \mathrm{~g} \cdot \mathrm{L}^{-1}$ ) N. oculata was centrifuged, using an industrial batch centrifuge, at Necton facilities. Algal paste was frozen at $-20{ }^{\circ} \mathrm{C}$. To remove rests of seawater 
salt, a portion of the algal paste was resuspended in $10 \mathrm{ml}$ ammonium formate solution $(0.5 \mathrm{M})$ and centrifuged for $15 \mathrm{~min}$ at $4400 \times g\left(\right.$ Beckman Avanti $\left.{ }^{\mathrm{TM}} \mathrm{J}-25\right)$. The rinsing procedure was repeated twice. Small portions of algal pellets were stored frozen $\left(-20{ }^{\circ} \mathrm{C}\right)$ prior to either oven drying $\left(60{ }^{\circ} \mathrm{C}\right.$, May 2007 and Jan 2008) or freeze-drying.

Total lipids were extracted from freeze-dried algal samples according to the Folch method [43] and FA profiles were determined using gas chromatography (GC) at Ifremer, Nantes, France. An aliquot of lipid was evaporated under nitrogen and trans-methylated by contact with methanol-sulphuric acid (98:2, v/v) in excess at $50^{\circ} \mathrm{C}$ overnight. After cooling, $2 \mathrm{~mL}$ of hexane and $1 \mathrm{~mL}$ of water were added and vortexed. The upper organic phase containing fatty acid methyl esters (FAMEs) was collected and assayed by gas chromatography using a PerkinElmer Autosystem equipped with an FID detector. Separation was done using helium as carrier gas on a fused-silica column (BPX-70, $60 \mathrm{~m}$ long, $0.25 \mathrm{~mm}$ i.d., $0.25 \mu \mathrm{m}$ film thickness, SGE) programmed from $55{ }^{\circ} \mathrm{C}$ (for $2 \mathrm{~min}$ ) to $150{ }^{\circ} \mathrm{C}$ at $20^{\circ} \mathrm{C} \cdot \mathrm{min}^{-1}$ then to $230{ }^{\circ} \mathrm{C}$ at $1.5^{\circ} \mathrm{C} \cdot \mathrm{min}^{-1}$. Sample was injected with a programmable split/splitless inlet and large-volume injection system (PSS) using the following temperature programme: $55^{\circ} \mathrm{C}$ (for $2 \mathrm{~min}$ ) to $350{ }^{\circ} \mathrm{C}$ at $200{ }^{\circ} \mathrm{C} \cdot \mathrm{min}^{-1}$. FAMEs were identified by comparison of their equivalent chain length with those of authentic standards. Quantification was done using margaric acid (C17:0) as internalstandard.

The neutral lipid class distribution-MAGs, DAGs, TAGs, FFA and oligomers - was determined using Gel Permeation Chromatography (GPC) method with Refractive Index (RI) detection (in-house designed GPC) at Neste Oil, Espoo, Finland. Samples were extracted with chloroform-methanol and chlorophyll was removed by Solid Phase Extraction prior to injection to GPC. Separation was done using isocratic elution with tetrahydrofuran $\left(1.0 \mathrm{~mL} \cdot \mathrm{min}^{-1}\right)$ as eluent, and three $50,100,500 \AA$ Agilent PLgel columns $(5 \mu \mathrm{m} 300 \times 7.5 \mathrm{~mm})$ in series.

Statistical analyses were performed using R (version 2.12.0, R Foundation for Statistical Computing, Vienna, Austria). Simple and multiple linear regressions were carried out to estimate the relationship between the dependent variables total lipids, FA and different lipid classes and the independent variables light as total global radiation (TGR) and temperature. TGR and temperature were obtained from the Meteorological Institute of Portugal (Instituto de Meteorologia). TGR and temperature data used for statistical analyses were integrated values of 10 consecutive days prior to sampling. The assumptions of normality of dependent variables were tested by Shapiro Wilk's test for normality and linearity was checked by plotting residuals $v s$. fitted values for each statistical model. Upon violation of these assumptions data were log-transformed (alt. squared or square root), however, with no significant effect on the statistical result.

\section{Conclusions}

Concerning the use of microalgae as feedstock for biofuel production (FAME biodiesel and HVO), our results address important issues about biomass and lipid yield and quality. In outdoors algal cultivation systems, a large portion of the algal biomass and lipid yield can be explained by seasonal variation in temperate latitudes. Yet, inter- and intra-seasonal variability in lipid content and composition can account up to $50 \%$ of the changes in controlled closed systems located in Southern Portugal. Calculations of oil yield production need to account for this high variability even when 
manipulating nutrient supply to boost algal lipid synthesis. The relative lipid increase may be constant over the year, but the real oil yield will differ widely at certain periods of the year due to a combination of seasonal biomass production, and intra-seasonal and diurnal variability. Summer and autumn were the favorable seasons for production of the most suitable FA for feedstock in biodiesel production.

\section{Acknowledgments}

We are grateful to Necton's technical staff for their help during the sampling period. Funding was provided by Neste Oil (Finland), the French Research Institute for Exploration of the Sea (Ifremer) and the Faculty of Natural Sciences and Technology, Linnæus University, Sweden. This is a contribution to the LnU Centre for Ecology and Evolution using Microbial models Systems (EEMiS).

\section{References}

1. Chisti, Y. Biodiesel from microalgae. Biotechnol. Adv. 2007, 25, 294-306.

2. Hu, Q.; Sommerfeld, M.; Jarvis, E.; Ghirardi, M.; Posewitz, M.; Seibert, M.; Darzins, A. Microalgal triacylglycerols as feedstocks for biofuel production: Perspectives and advances. Plant J. 2008, 54, 621-639.

3. Schenk, P.M.; Thomas-Hall, S.R.; Stephens, E.; Marx, U.C.; Mussgnug, J.H.; Posten, C.; Kruse, O.; Hankamer, B. Second generation biofuels: High-efficiency microalgae for biodiesel production. Bioenergy Res. 2008, 1, 20-43.

4. Meng, X.; Yang, J.; Xu, X.; Zhang, L.; Nie, Q.; Xian, M. Biodiesel production from oleaginous microorganisms. Renew. Energy 2009, 34, 1-5.

5. Renaud, S.M.; Parry, D.L.; Thinh, L.V.; Kuo, C.; Padovan, A.; Sammy, N. Effect of light-intensity on the proximate biochemical and fatty-acid composition of Isochrysis sp. and nannochloropsis-oculata for use in tropical aquaculture. J. Appl. Phycol. 1991, 3, 43-53.

6. Dhert, P.; Rombaut, G.; Suantika, G.; Sorgeloos, P. Advancement of rotifer culture and manipulation techniques in Europe. Aquaculture 2001, 200, 129-146.

7. Rodolfi, L.; Zittelli, G.C.; Barsanti, L.; Rosati, G.; Tredici, M.R. Growth medium recycling in Nannochloropsis sp. mass cultivation. Biomol. Eng. 2003, 20, 243-248.

8. Roncarati, A.; Meluzzi, A.; Acciarri, S.; Tallarico, N.; Melotti, P. Fatty acid composition of different microalgae strains (Nannochloropsis sp., Nannochloropsis oculata (Droop) Hibberd, Nannochloris atomus Butcher and Isochrysis sp.) according to the culture phase and the carbon dioxide concentration. J. World Aquacult. Soc. 2004, 35, 401-411.

9. Bentley, C.D.; Carroll, P.M.; Watanabe, W.O.; Riedel, A.M. Intensive rotifer production in a pilot-scale continuous culture recirculating system using nonviable microalgae and an amonia neutralizer. J. World Aquacult. Soc. 2008, 39, 625-635.

10. Sandnes, J.M.; Källqvist, T.; Wenner, D.; Gislerød, H.R. Combined influence of light and temperature on growth rates of Nannochloropsis oceanica: Linking cellular responses to large-scale biomass production. J. Appl. Phycol. 2005, 17, 515-525.

11. Hu, C.; Li, M.; Li, J.; Zhu, Q.; Liu, Z. Variation of lipid and fatty acid compositions of the marine microalga Pavlova viridis (Prymnesiophyceae) under laboratory and outdoor culture conditions. World J. Microbiol. Biotechnol. 2007, 24, 1209-1214. 
12. Zittelli, G.C.; Lavista, F.; Bastianini, A.; Rodolfi, L.; Vincenzini, M.; Tredici, M.R. Production of eicosapentaenoic acid by Nannochloropsis sp. cultures in outdoor tubular photobioreactors. J. Biotechnol. 1999, 70, 299-312.

13. Rodolfi, L.; Chini Zittelli, G.; Bassi, N.; Padovani, G.; Biondi, N.; Bonini, G.; Tredici, M.R. Microalgae for oil: Strain selection, induction of lipid synthesis and outdoor mass cultivation in a low-cost photobioreactor. Biotechnol. Bioeng. 2009, 102, 100-112.

14. Chiu, S.Y.; Kao, C.Y.; Tsai, M.T.; Ong, S.C.; Chen, C.H.; Lin, C.S. Lipid accumulation and $\mathrm{CO}_{2}$ utilization of Nannochloropsis oculata in response to $\mathrm{CO}_{2}$ aeration. Bioresour. Technol. 2009, 100, 833-838.

15. Gouveia, L.; Oliveira, A.C. Microalgae as a raw material for biofuels production. World J. Ind. Microbiol. Biotechnol. 2009, 36, 269-274.

16. Sukenik, A.; Yamaguchi, Y.; Livne, A. Alterations in lipid molecular-species of the marine eustigmatophyte Nannochloropsis sp. J. Phycol. 1993, 29, 620-626.

17. Brown, M.R.; Dunstan, G.A.; Norwood, S.J.; Miller, K.A. Effects of harvest stage and light on the biochemical composition of the diatom Thalassiosira pseudonana. J. Phycol. 1996, 32, 64-73.

18. Berge, J.P.; Gouygou, J.P.; Dubacq, J.P.; Durand, P. Reassessment of lipid-composition of the diatom, skeletonema-costatum. Phytochemistry 1995, 39, 1017-1021.

19. Tonon, T.; Harvey, D.; Larson, T.R.; Graham, I.A. Long chain polyunsaturated fatty acid production and partitioning to triacylglycerols in four microalgae. Phytochemistry 2002, 61, 15-24.

20. Sukenik, A.; Carmeli, Y.; Berner, T. Regulation of fatty-acid composition by irradiance level in the eustigmatophyte Nannochloropsis sp. J. Phycol. 1989, 25, 686-692.

21. Suen, Y.; Hubbard, J.S.; Holzer, G.; Tornabene, T.G. Total lipid production of the green-alga Nannochloropsis sp. qii under different nitrogen regimes. J. Phycol. 1987, 23, 289-296.

22. Takagi, M.; Watanabe, K.; Yamaberi, K.; Yoshida, T. Limited feeding of potassium nitrate for intracellular lipid and triglyceride accumulation of Nannochloris sp UTEX LB1999. Appl. Microbiol. Biotechnol. 2000, 54, 112-117.

23. Weldy, C.S.; Huesemann, M. Lipid production by Dunaliella Salina in batch culture: Effects of nitrogen limitation and light intensity. J. Undergrad. Res. 2007, 115-122.

24. Takagi, M.; Karseno; Yoshida, T. Effect of salt concentration on intracellular accumulation of lipids and triacylglyceride in marine microalgae Dunaliella cells. J. Biosci. Bioeng. 2006, 101, 223-226.

25. Sukenik, A.; Bennett, J.; Mortain-Bertrand, A.; Falkowski, P.G. Adaptation of the photosynthetic apparatus to irradiance in Dunaliella tertiolecta: A kinetic study. Plant Physiol. 1990, 92, 891-898.

26. Fabregas, J.; Maseda, A.; Dominguez, A.; Otero, A. The cell composition of Nannochloropsis sp. changes under different irradiances in semicontinuous culture. World J. Microbiol. Biotechnol. 2004, 20, 31-35.

27. Lynch, D.V.; Thompson, G.A. Low temperature-induced alterations in the chloroplast and microsomal-membranes of Dunaliella salina. Plant Physiol. 1982, 69, 1369-1375.

28. Thompson, P.A.; Guo, M.X.; Harrison, P.J.; Whyte, J.N.C. Effects of variation in temperature. 2. On the fatty-acid composition of 8 species of marine-phytoplankton. J. Phycol. 1992, 28, 488-497. 
29. Aatola, H.; Larmi, M.; Sarjovaara, T. Hydrotreated Vegetable Oil (HVO) as a renewable diesel fuel: Trade-off between $\mathrm{NO}_{x}$, particulate emission, and fuel consumption of a heavy duty engine. SAE Int. J. Engines 2009, 1, 1251-1262.

30. Herrera-Valencia, V.A.; Contreras-Pool, P.Y.; Lopez-Adrian, S.J.; Peraza-Echeverria, S.; Barahona-Perez, L.F. The green microalga chlorella saccharophila as a suitable source of oil for biodiesel production. Curr. Microbiol. 2011, 63, 151-157.

31. Knothe, G. "Designer" biodiesel: Optimizing fatty ester composition to improve fuel properties. Energy Fuels 2008, 22, 1358-1364.

32. Ramos, M.J.; Fernandez, C.M.; Casas, A.; Rodriguez, L.; Perez, A. Influence of fatty acid composition of raw materials on biodiesel properties. Bioresour. Technol. 2009, 100, 261-268.

33. Liu, Y.; Sotelo-Boyás, R.; Murata, K.; Minowa, T.; Sakanishi, K. Hydrotreatment of vegetable oils to produce bio-hydrogenated diesel and liquefied petroleum gas fuel over catalysts containing sulfided Ni-Mo and solid acids. Energy Fuels 2011, 25, 4675-4685.

34. Renaud, S.M.; Thinh, L.V.; Lambrinidis, G.; Parry, D.L. Effect of temperature on growth, chemical composition and fatty acid composition of tropical Australian microalgae grown in batch cultures. Aquaculture 2002, 211, 195-214.

35. Israelachvili, J.N.; Marcelja, S.; Horn, R.G. Physical principles of membrane organization. Q. Rev. Biophys. 1980, 13, 121-200.

36. Fisher, T.; Berner, T.; Iluz, D.; Dubinsky, Z. The kinetics of the photoacclimation response of Nannochloropsis sp. (Eustigmatophyceae): A study of changes in ultrastructure and PSU density. J. Phycol. 1998, 34, 818-824.

37. Hoekman, S.K.; Broch, A.; Robbins, C.; Ceniceros, E.; Natarajan, M. Review of biodiesel composition, properties, and specifications. Renew. Sustain. Energy Rev. 2012, 16, 143-169.

38. Dunstan, G.A.; Volkman, J.K.; Barrett, S.M.; Garland, C.D. Changes in the lipid-composition and maximization of the polyunsaturated fatty-acid content of 3 microalgae grown in mass-culture. J. Appl. Phycol. 1993, 5, 71-83.

39. Shanklin, J.; Cahoon, E.B. Desaturation and related modifications of fatty acids. Annu. Rev. Plant Phys. 1998, 49, 611-641.

40. Volkman, J.K.; Brown, M.R.; Dunstan, G.A.; Jeffrey, S.W. The biochemical-composition of marine microalgae from the class eustigmatophyceae. J. Phycol. 1993, 29, 69-78.

41. European Standard EN 14214. Automotive fuels-Fatty acid methyl esters (FAME) for diesel engines_-Requirements and test methods. AFNOR: Saint-Denis, France, 2004.

42. Wijffels, R.H.; Barbosa, M.J.; Eppink, M.H.M. Microalgae for the production of bulk chemicals and biofuels. Biofuels Bioprod. Biorefin. 2010, 4, 287-295.

43. Folch, J.; Lees, M.; Stanley, G.H.S. A simple method for the isolation and purification of total lipides from animal tissues. J. Biol. Chem. 1957, 226, 497-509.

(C) 2012 by the authors; licensee MDPI, Basel, Switzerland. This article is an open access article distributed under the terms and conditions of the Creative Commons Attribution license (http://creativecommons.org/licenses/by/3.0/). 